\title{
ANÍBAL BASCUÑÁN Y EL PENSAMIENTO ADMINISTRATIVO HISPANOAMERICANO
}

\section{ANÍBAL BASCUÑÁN AND HISPANIC AMERICAN ADMINISTRATIVE THINKING}

\author{
OMAR GUERRERO OROZCO \\ Universidad Nacional Autónoma de México, México \\ omarguerrer@yahoo.com
}

\section{RESUMEN}

El artículo estudia el pensamiento administrativo de Aníbal Bascuñan Valdés, a partir de un análisis de su libro Elementos de Ciencia de la Administración Pública (1963). Se destaca el valor de Bascuñán en la historia intelectual de la disciplina, por ser el primer pensador hispanoamericano en sustentar sin titubeos el estatuto autónomo de la administración pública y por concebirla como conocimiento científico bajo un enfoque óntico, donde su ser está dotado de tipicidad y es susceptible de desarrollar principios. Se concluye que la de Bascunán es una de las obras más avanzadas dentro de la disciplina durante la década de 1960, sobre todo por considerar a la administración pública como ciencia autónoma y como ciencia social.

Palabras clave: Administración pública, Teoría de la administración pública, Historia intelectual, Pensamiento latinoamericano.

\begin{abstract}
The article examines the administrative thinking of Aníbal Bascunán Valdés, derived from the analysis of his book Elementos de Ciencia de la Administración Pública (1963). It highlights the value of Bascunanan to the intellectual history of the discipline, for being the first Hispanic American thinker who without hesitation supported the autonomous status of public administration and for conceiving it as scientific knowledge under an ontic approach, where his being is endowed with an identity and able to develop principles. It is concluded that the work of Bascunan is one of the most developed within the discipline during the 1960s, above all because it considers public administration as an autonomous science and social science.
\end{abstract}

Keywords: Public administration, Public administration theory, Intellectual history, Latin American thinking.

Revista de Gestión Pública

VOLUMen I, Número 1

ISSN 0719-1820

pp. $55-68$ 


\section{INTRODUCCIÓN}

Cuando optamos por titular este artículo como: "Aníbal Bascunán y el Pensamiento Administrativo Hispanoamericano", consideramos de entrada que ese eminente personaje hoy en día es desconocido en la mayor parte de Hispanoamérica. Este hecho infortunado nos movió a presentar un texto de reivindicación de su obra, y con afán resaltar su relevancia dentro del pensamiento administrativo. Y si, en efecto, nos centramos en nuestros países, también nos propusimos extender sus ideas dentro de la ciencia de la administración pública mundialmente considerada. No obstante, debemos dar énfasis al hecho de que Bascunán es conocido y reconocido en su tierra natal, Chile, como lo atestigua un ensayo aquí consultado (Drapkin 2004).

Así pues, un problema destacable es el sitio de Bascuñan fuera de su país, y especialmente en Hispanamérica. Quien escribe estas líneas, que ha tenido un apetito intelectual singular por la búsqueda y encuentro de pensadores administrativos que se remonta a cuatro décadas atrás, tuvo noticia del libro del Doctor Bascuñan recientemente, y lo recibió con una emocionante sorpresa. En efecto, la obra fue conocida por mi persona en julio de 2008 -merced a la casualidad, así como el uso de la Internet- y su encuentro significó un hallazgo extraordinario por su valor científico, y su significado pasado y presente

Aníbal Bascunán nació en Santiago de Chile en noviembre 4 de 1905, realizando estudios jurídicos en su país, España, Francia e Italia. Bajo la docta mano de Rafael Altamira se graduó con un trabajo sobre la organización imperial inca, toda vez que fue un profesor insigne de la cátedra titulada "Ciencia de la Administración Pública”, fundada por su persona en la Escuela de Ciencias Políticas y Administrativas (establecida en 1954), en la que dirigió su Instituto que llevó por nombre a las mismas materias (Drapkin 2004). De las lecciones en aula surgió su opera magna: Elementos de Ciencia de la Administración Pública, publicada a mediados de 1963. Bascuñán murió en diciembre 14 de 1988.

Particularmente situaremos el estudio del pensamiento administrativo de Bascuñán en la década de 1960, cuando se publicaron otras dos obras muy valiosas emanadas de la pluma de José Galván Escobedo y Wilburg Jiménez Castro, época en la cual se traducen al español los libros que entonces significaron un decenio de oro del pensamiento administrativo. Naturalmente, haremos un breve ejercicio de análisis de la teoría administrativa de Bascunán que situaremos en la década mencionada, así como hacia el presente. 


\section{LA DÉCADA DE 1960}

A lo largo de la década de 1960 se produjeron buena parte de los libros más relevantes en administración pública, escritos por autores hispanoamericanos. El camino había sido inaugurado por la obra Introducción a la Administración Pública del puertorriqueño Pedro Muńoz Amato, un texto en dos tomos publicado en 1954 y 1957. Y si bien es cierto que el libro es muy anterior a los publicados en el decenio siguiente, también lo es que fue el modelo que inspiró los escritos posteriores.

Ya situados en la década mencionada, el primer producto es el Tratado de Administración General del mexicano José Galván Escobedo, egresado de imprenta en 1962. Un americanismo incipiente distingue a ambos trabajos, pues el primero se publicó en México y el segundo en Costa Rica. Siguió otra obra trascendental: Introducción al Estudio de la Teoría Administrativa del costarricence Wilburg Jiménez Castro, que apareció en México en 1963. Todas estas obras tuvieron la virtud de que, en su tiempo, fueron un buen camino para la superación del formalismo con que se enseñaba la administración pública en las universidades de Hispanoamérica, circunstancia que le consta a nuestra persona, que pudo observar la paradoja de un plan de estudios en administración pública sin fuentes de la especialidad y libros de la materia fuera de los curriculum de la especialidad. El tercer libro es Elementos de Ciencia de la Administración Pública de Aníbal Bascuñán, publicado en 1963 en Santiago de Chile. En contraste con las obras de Galván Escobedo y Jiménez Castro, ampliamente difundidas, parece ser que el libro se limitó a la órbita chilena y no tuvo trascendencia internacional, lo cual es un infortunio para nuestra disciplina, y es algo que debemos enmendar.

Tampoco ha existido, hasta el presente, una década posterior al decenio de 1960 donde sea visible una pléyade de pensadores administrativos accesibles a los lectores a través de traducciones realizadas en España, México, Argentina, Puerto Rico y Costa Rica ${ }^{1}$. Entonces, ese acceso bibliográfico acercó obras distantes en el tiempo como el libro de Leonard White, Introducción al Estudio de la Administración Pública, publicado originalmente en 1926 y traducido en 1964. Antes, empero, ya era accesible la versión puertorriqueña del texto de Herbert Simon y sus colegas, Administración Pública, traducido en 1956 (seguido de la versión mexicana de 1968, la cual, dicho sea de paso, es diversa a la edición caribeña). Fue pues, por medio de las versiones en castellano, que en ese decenio fue posible consultar al más grande pensador estadounidense, Dwight Waldo, quien en 1948 produjo su opera magna, vertida en 1961 a nuestro idioma como Teoría Política de la Administración Pública. Otro de sus libros cimeros, Estudio de la Administración Pública, se tradujo en 1964, así como su Administración Pública en 1967.

\footnotetext{
En el segundo anexo bibliográfico se hace una relación puntual de las fuentes analizada enseguida.
} 
Debemos destacar la edición de un texto simbólico de entonces, ampliamente consultado, que fue producido por la ONU (1962) con el título de Manual de Administración Pública. El escrito fue un botón de muestra de un tema emergente relativo a la modernización administrativa, en la que destacó el libro de Harold Alderfer, La Administración Pública en las Nuevas Naciones, publicado en 1970. En fin, una más de las obras representativas es Burocracia y Desarrollo Político, un volumen colectivo a cargo de Joseph LaPalombara (1970) que tuvo una extensa difusión y se consultó intensamente como material de enseñanza. Asimismo se debe resaltar la doble traducción al español de la obra cumbre del gerencialismo de los años de 1930, que fue puesto en manos de lectores hispanos e hispanoamericanos. Se trata del texto colectivo capitaneado por Luther Gulick y Lyndall Urwick, Ensayos sobre la Ciencia de la Administración, editada primero en Costa Rica (1970) y después en España (1973).

Desde una perspectiva diversa, un escrito de rompimiento epistemológico de mucho crédito, el mejor libro de Herbert Simon, El Comportamiento Administrativo, se publicó en España en 1964. Sus aportes al estudio de las organizaciones es tan relevante como el texto célebre de Chester Barnard, obra principal, vertido confusamente con el título de Las Funciones de los Elementos Dirigentes y editado asimismo en Espańa en 1959. Igualmente debemos considerar el texto de Amitai Etzioni, Organizaciones Modernas, egresado de imprenta en 1965. James March se asoció con Simon en la obra Teoría de la Organización, traducida en 1969.

En fin, en Argentina se tradujo tempranamente la obra principal de James Burnham, La Revolución de los Directores, aparecida originalmente en 1943 y reeditada en 1962 y 1967. En fin, del gran pensador británico Herman Finer se vertió al español su obra Teoría y Práctica del Gobierno Moderno, publicada en España en 1964.

\section{EL PENSAMIENTO HISPANOAMERICANO EN LA DÉCADA DE 1960}

El pensamiento administrativo hispanoamericano se remonta al siglo XIX (e incluso en XVIII), pero no debemos regresar demasiado para situarnos en nuestra época, pues la década mencionada tiene su tipicidad, y es visible su punto de arranque un lustro atrás con la obra del puertorriqueño Pedro Muñoz Amato, como lo hicimos saber. Uno de los rasgos de esa tipicidad es el problema de definición de la disciplina, punto que traslada el problema a su diferenciación con los negocios privados, así como a la dicotomía política-administración, que estaban en boga en los Estados Unidos y de allí se importaron a Hispanoamérica. Es decir, que aquí en los años de 1960 se importaron problemas epistemológicos inexistentes en la realidad. Sin embargo, desde la época de Muñoz Amato, esa problematización contribuyó a liberar el estudio de la administración pública del 
ámbito dominante del derecho administrativo, toda vez que se plantea como un tema de interés político. De aquí que ese autor explique que, en su sentido más amplio, la administración pública es el gobierno, toda vez que es todo el conjunto de conducta humana que determina cómo se distribuye y ejerce la autoridad política (Muñoz Amato 1954 I: 15, 24 y 26). En efecto, asume que se trata de un ámbito de la ciencia política. En resumen, Muñoz Amato apunta que la administración pública es la fase del gobierno relativa a la ordenación cooperativa de personas mediante la planificación, organización, educación y dirección de su conducta, con el propósito de realizar los fines del sistema político. Las fuentes de Muñoz Amato son sólo los autores estadounidenses, muchos de ellos mencionados páginas arriba

Muńoz Amato es el punto de partida, pero no de manera lineal, sino condicional, como es visible directamente en Bascunán, así como con variaciones sensibles en Galván Escobedo y Jiménez Castro, más influenciados por el gerencialismo estadounidense.

Como lo adelantamos, el Tratado de Administración General del mexicano José Galván Escobedo, publicado en Costa Rica en 1962, fue el primero de los textos relevantes en la década mencionada. Se trata de un texto muy útil que respondió a lo que entonces exigía la vida académica para llenar las currícula de los programas de las asignaturas de administración pública. Pero ello fue principalmente porque representó una alternativa a los estudios de derecho administrativo, otrora dominantes. El libro refleja fielmente un enfoque que se aleja del planteamiento de Muñoz Amato, pues en sus páginas predomina un concepto de administración como una disciplina única e indivisible, una ciencia que no distingue entre lo público y lo privado; el libro implica, como lo enuncia su título, un tratado de "administración general". Al mismo tiempo, su autor manifiesta claramente el abordar un objeto de estudio científico merced a que la administración, como proceso, es universal. De modo que se le encuentra en todos los tiempos y en todos los lugares porque no existe, ni existió, grupo humano que no implique un concepto administrativo expresa o tácitamente (Galván Escobedo 1963: XV). Asimismo, la administración es un producto social que se encuentra sujeta al tiempo y las condiciones cambiantes; y sus principios son válidos solamente cuando se adaptan y surgen como resultado de las condiciones privativas en el tiempo y el lugar en que tratan de aplicarse. En suma, existe una teoría general que se puede denominar indistintamente "administración" o "gerencia", cuyo objeto es estudiar los procesos administrativos que son esenciales en cualquier situación, en la cual un grupo de individuos se organiza para lograr propósitos comunes.

Entrando al tema de la administración pública, Galván Escobedo anota que ella aún no es una ciencia "completa", si bien tiene conocimientos sistemáticos 
basados en principios relativamente generales. Por consiguiente, se deben distinguir la administración pública y la administración privada, pues la primera es la ejecución y observancia de la política gubernamental y le atañen los poderes, organización y técnicas relacionados con la aplicación de esa política (Galván Escobedo 1963: 10-13). En efecto, "la administración pública es la ley en acción, es la parte ejecutiva del gobierno". Opuestamente, corresponden a la administración privada las actividades de los particulares en general, ya se trate de la industria que es su campo fundamental, ya lo sea de la iglesia, las universidades o instituciones de beneficencia. Hay que añadir un tercer tipo, la administración mixta o cuasi pública que está bajo la jurisdicción compartida del gobierno y los particulares. Por lo tanto, desde el punto de vista de los organismos en particular, la administración es de tres clases, pero se trata de una disciplina única con principios generales para ese trío.

Wilburg Jiménez Castro publica su obra un año después del trabajo de Galván Escobedo. Como su antecesor, también argumento a favor de una administración única. En su entender, la administración se puede definir de manera unívoca para fines didácticos, incluyendo sólo los aspectos más importantes. Esto es: la administración es una ciencia social integrada por principios, técnicas y prácticas, cuya aplicación a las comunidades humanas facilita estab1ecer sistemas racionales de esfuerzo cooperativo por medio de los cuales se pueden alcanzar propósitos comunes, que no es factible alcanzar individualmente (Jiménez Castro 1963: 20, 27-28). Asimismo, Jiménez Castro enfatiza la universalidad de la administración, motivo por el cual recomienda se eliminen las fronteras en el uso de los principios administrativos porque es la que le da una extraordinaria versatilidad a su aplicación en "muy variadas condiciones y latitudes". Ella es, citando a Charles A. Beard, "la ciencia llave de la civilización contemporánea". Asimismo, esa universalidad de la administración se prueba también merced a la aplicación de sus principios válidamente y por igual en las empresas privadas y en las públicas.

De aquí que la administración pública y la administración privada son dos "ramas especializadas" de la misma disciplina, emanando de ello que sus principios tengan muchas bases científicas comunes. "Ambas son partes de un todo". De manera que, como disciplinas, técnicas y artes, no difieren desde un punto de vista teórico y en la práctica su separación no es tan tajante como se piensa (Jiménez Castro 1963: 28-29). Al respecto Jiménez Castro recurre a la comprobación que ofrece en el análisis de los objetivos sociales y de las estructuras organizativas, la programación de labores y las normas aplicables para la administración del personal, entre otros. Asimismo, sus diferencias son más bien circunstanciales ("de funciones o de campos de actividad"), así como por el tamaño de las organizaciones. Por lo tanto, bajo condiciones normales, es probable encontrar más similitudes y problemas comunes, que diferencias. Habida cuenta de estos argumentos, Jiménez Castro obvia definir a una o la otra. 
Está de más insistir en el parentesco evidente de Galván Escobedo y Jiménez Castro, cuyo eje es la universalidad de la administración. Su énfasis en nuestro entender radica en el descubrimiento de la "administratividad" inherente a todos los grupos humanos, hecho que estimula e impulsa a los dos autores a identificarla como inherente a toda organización, por encima de sus diferencias. Esta perspectiva fue muy influyente en la década de 1960, y aún hoy en día tiene adeptos; pero el flujo predominante de la marea administrativa se desplazaba dentro del torrente de la administración pública como una disciplina especializada, como lo adelantó Muñoz Amato, y era patente en la mayor parte de la bibliografía traducida al castellano. La posición de Bascunán fue la misma.

\section{EL PENSAMIENTO ADMINISTRATIVO DE ANÍBAL BASCUÑÁN}

La lectura del libro de Aníbal Bascuñán nos inspira dos reflexiones, la primera de las cuales es que su difusión más allá de Chile desde tiempo atrás, habría producido enorme beneficio a las generaciones de entonces y habría repercutido de un modo beneficioso en el presente. La otra meditación es que, divulgando hoy mismo sus aportes, podremos colaborar a rendirle los créditos que merece, situarlo en el pensamiento administrativo hispanoamericano y mundial, y aprovechar su enorme sabiduría.

Por principio, debemos resaltar que Bascuñán estaba convencido de que la administración pública era en su época una ciencia independiente. De modo que se pregunta si debe hablarse en plural como ciencias administrativas, o en singular como ciencia administrativa; o bien ciencia de la administración. Su país y su plantel docente se mueven en dirección de la tradición europea. Esto explica la ruta intelectual de Bascunán como diversa a la seguida por Galván Escobedo y Jiménez Castro, más apegados al pensamiento administrativo estadounidense (Bascuñán Valdés 1963: 80-81). De ese punto emana su perspectiva sobre la administración pública como parte de las ciencias sociales y de las humanidades, y no como una rama de una disciplina administrativa universal. En efecto, la tradición europea es observable en sus fuentes documentales ${ }^{2}$, donde destaca Georges Langrod (1954), André Molitor (1958), y Pietro Gasparri (1959). Al mismo tiempo, el énfasis de la pertenencia de la administración pública a las disciplinas referidas implica una toma de distancia tácita del llamado enfoque "administrativo científico", especialmente del taylorismo cuyos procedimientos de estudio y aplicación observa más próximos a las ciencias físico-matemáticas. Bascuñán recuerda que la ciencia de la administración pública es la que tiene la historia más remota, incluso paralela a la formación del Estado moderno, mientras que el "administrativismo científico" emerge a comienzos del siglo XX con los trabajos del francés Henri Fayol, el estadounidense Frederick Taylor, y el británico Lyndall Urwick.

2 En su escrito, Aníbal Bascunán advierte que por razones de urgencia no pudo preparar técnicamente las referencias bibliográficas, motivo por el cual no pudimos realizar una exploración más amplia de las fuentes que utilizó. 
Comprometido del todo en pro de la administración pública como disciplina científica, Bascuñán reprocha a Muñoz Amato que se resista a aceptar la existencia de una ciencia de la administración pública, cuando así lo merece, considerando principalmente su contenido y sus relaciones con las ciencias sociales y las humanidades. Lo mismo achaca a Wilburg Jimenez Castro, quien en un curso sobre administración sustentado en Montevideo alude más bien un "arte científico" (Bascunán Valdés 1963: 81-82). Habida cuenta de sus fuentes europeas, Bascunán apela a Langrod, para quien la doctrina alemana asigna la mayor jerarquía a la ciencia de la administración y brinda un papel auxiliar a otras disciplinas complementarias, como el derecho administrativo y la teoría administrativa. Sobre esta base Bascuñán asume la distinción entre ciencia de la administración en sentido genérico (verwaltungswissenchaft) y teoría administrativa en sentido estricto (Verwaltlehre), esta última como parte de la primera. Bascuñán quizá sea el primer pensador administrativo hispanoamericano quien proclama, sin reservas, que la "ciencia de la administración pública ha logrado una plena autonomía"

Obviamente, Aníbal Bascuñán discrepa con quienes observan a la administración pública como simple suma o agregado de temas provenientes de diversas zonas ónticas (óntica: "lo que es", "el ser") como el derecho, política, economía, administración general, sociología, sicología o historia. Por el contrario, se trata de un objeto complejo, pero unitario, al cual se arriba conceptualmente con procesos intelectuales como ocurre con las ciencias que conciernen al hombre, arriba referidas. Planteado el problema epistemológico, Bascunán se adentra en el tema metodológico donde apunta que la disciplina relativa a la administración pública, cuyo empeño se encamina a desentrañar su naturaleza sin desfiguramientos ni falsificación, emplea un método ("o mejor, una metodología") propio del objeto que reclama su individualidad óntica; un objeto cuyo centro de interés que parte del problema que Bascuñán sintetiza en la cuestión sobre “qqué es administración pública?” La respuesta se halla situada en su plano equidistante de todas las disciplinas que colaboraron al nacimiento de la administración pública o que extendieron su alcance.

Para esquematizar su tesis, Bascuñán recurre a una figura sumamente ilustrativa sobre el plano de la ciencia de la administración pública, situada en un círculo central, mientras que en la periferia se mueven ocho disciplinas que asimismo ocupan cada cual su aro: historia; derecho administrativo; ciencia política; ciencia administrativa; economía; sociología; psicología y antropología cultural. Una vez articulados los aros periféricos por medio del círculo central, surge un magno concierto en el cual las zonas traslapadas implican ámbitos bi-disciplinarios con la administración pública:

- Historia (historia de la administración pública).

- Derecho administrativo (ordenamiento jurídico de la administración pública). 
- Ciencia política (política administrativa pública).

- Ciencia administrativa (administración del sector público. Economía (administración financiera).

- Sociología (administración comunitaria).

- Psicología (interacciones administrativas públicas).

- Antropología cultural (administración pública en cuanto objetivación de la cultura).

Pero la administración pública tiene su propia tipicidad, es decir, rasgos que le identifican y caracterizan de entre otros fenómenos sociales, que en letra de Bascuñán refiere su contenido esencial. De aquí deriva la división de tres posturas: la primera se refiere a "administración a secas" y reposa en un solo elemento, la conducta humana coordinada (Bascuñán Valdés 1963: 105). Se trata de un elemento subjetivo. La segunda postura vincula los elementos subjetivo y objetivo, y se refiere a la estructura u organismo de la administración. La tercera concepción se identifica solamente con el elemento objetivo, y se trata de las bases orgánicas de la administración pública. Los administrativistas puros se inclinan por la primacía, y aún más, por la exclusividad del elemento subjetivo, mientras que el "administrativismo jurídico" opta por la coexistencia de un corpus y una función, elementos "copulativamente" integrantes del contenido de la administración pública. Bascuñán opta por la demostración teórica y la comprobación práctica de la conjunción de elementos objetivos y subjetivos, es decir, por una estructura real y concreta que emana como producto socio-económico-cultural, así como de la "conducta coordinada y normada moral, jurídica y técnicamente" de los funcionarios que integran los órganos de dicha estructura. En efecto, integran la organización sustantiva de la administración pública el personal administrativo (el funcionariado público), el patrimonio público (bienes inmuebles y muebles y fondos presupuestarios) y el sistema normativo.

Una vez establecidos los cimientos epistemológicos, Bascunán está en disposición de ofrecer una definición:

Nosotros diríamos — parodiando el lenguaje marxista — que la administración pública es la actividad administrativa acumulada; si se nos permite la expresión, es la capitalización del trabajo administrativo que, en la medida de su crecimiento material y humano y de la intensificación de su misión interventora en pro del bienestar colectivo —último fin de toda administración pública-, determina una complejidad creciente y cada vez más especializada de la función administrativa, estructurada en órganos y servicios públicos (Bascuñán Valdés 1963: 105-106). 
La administración pública es definida de un modo no sólo singular, sino magistral. El pensamiento administrativo de Bascuñán tiene asimismo un corolario excelso en la definición de la administración pública, en la cual armoniza una visión evolutiva (acumulativa) de la función pública, que se perfecciona, que se convierte en institución y que forja al servidor público profesional.

\section{ANÁLISIS ONTOLÓGICO DE LA ADMINISTRACIÓN PÚBLICA}

Aníbal Bascunán se propuso hacer un examen ontológico de la administración pública, es decir, examinar su ser partiendo de la pregunta ya referida: ¿qué es administración pública?, considerada como su unidad ontológica, cuyo centro de interés se encuentra fuera de cada una de las restantes ciencias sociales. En efecto, la ontología es el estudio de los supuestos que podemos entender, esto es, de algo que rigurosamente se llama administración pública y que puede ser conocida. Ese conocimiento asume que existen ciertas acciones, intenciones y conductas humanas, así como prácticas y estructuras, que "existen" (White, 1999: 11). En aquí donde Bascuñán encuentra el sustento de una "ciencia social autónoma", denominada ciencia de la administración pública. Bajo el examen ontológico, la administración pública representa un complejo de elementos situado "en y para" la comunidad porque es la sociedad la que la crea y mantiene, y es en pro de la satisfacción de las necesidades colectivas que la administración pública se mueve (Bascuñán Valdés, 1963: 110-111). Del complejo de esos elementos, Bascunán separa los rasgos que son estructurales (bajo una consideración estática) y el elemento funcional perceptible en su dinámica; y que corresponden respectivamente a los elementos: objetivo y subjetivo ya referidos.

Los elementos estructurales (u objetivos) son el funcionariado público; el estatuto normativo (integrado por el sistema de normas jurídicas, técnico-administrativas, éticas y sociales); y el patrimonio público (bienes de cualquiera naturaleza, presupuestos, etc.). Por su parte, el elemento funcional se expresa en la dinámica de los tres elementos precedentes, bajo la presidencia del elemento "funcionariado público" (a través de sus agentes superiores); y se resuelve en el "elemento subjetivo"; es decir, en actividad pública o "función pública". Los servicios públicos especializadamente concretan la función administrativa para la satisfacción de las necesidades colectivas.

Bascuñán concluye que, si bien, como en el Fausto de Goethe, se pudiera decir que "en un principio fue la acción", con el correr del tiempo la función administrativa acumulada devino en administración pública. En efecto, refrenda que "la administración pública no es sino la capitalización del trabajo administrativo" (Bascuñán Valdés 1963: 113). Paralelamente, la función pública, que antes era el patrimonio y el deber (derecho de todo ciudadano), posteriormente se convirtió en el trabajo técnico-profesional del funcionario público que es quien anima la realización de los servicios públicos. 


\section{FUENTES INTELECTUALES DE LA OBRA DE BASCUÑÁN}

Además de los argumentos expuestos por Aníbal Bascunán en pro de un concepto autónomo y científico de la administración pública, se puede extender su bagaje bibliográfico como sustento de esos argumentos $\mathrm{y}$, asimismo, como sus fuentes intelectuales e ideológicas.

Bascuñán es un profesor de la materia muy bien dotado de capacidades retóricas, como suele ocurrir entre los docentes profesionales. Obviamente la retórica debe entenderse en su sentido clásico, es decir, como discurso persuasivo; y más propiamente en el sentido de "cómo razonar, cómo decir y cómo persuadir" (Hernandez Gil 1975: 16, 19-20). De aquí que la retórica ha estado muy vinculada con la gramática, literatura, lógica, dialéctica, jurisprudencia y la preparación de la normatividad administrativa. Es, en suma, el estudio de todas las formas de hacer cosas con las palabras (Majone 1989: 7-8). Y muchas son las cosas interesantes que Bascunán realiza con las palabras, pues asimismo es un escritor consumado por cuanto que persuade, al mismo tiempo que convence ${ }^{3}$.

En ese segundo momento relativo a la convicción, Bascunán convoca la fuerza probatoria del estado del arte de su tiempo, entonces plagado de grandes pensadores cuyas obras conoce y domina en plenitud. En sus citas encontramos obras monumentales del pensamiento administrativo europeo, como el escrito de André Molitor (1958) sobre la enseńanza de la administración pública y el de Pietro Gasparri (1959) sobre la ciencia de la administración. De George Langrod (1954) cita un curso de administración comparada, un tema central de entonces, así como una de sus obras más relevantes y trascendentales, aunque con menos difusión de la que merece, cuyo tema es el estudio de la administración pública en los Estados Unidos. Otro autor francés citado es Pierre Ecoubé, no tan conocido como Langrod, pero también muy destacado y reconocido en Hispanoamérica, principalmente en la ESAPAC, donde en 1955 profesó un curso introductorio administración pública que ha permanecido mecanografiado. La celebridad de Escoubé deriva de ser quien redescubrió a Charles-Jean Bonnin, fundador de la ciencia de la administración pública (Escoubé 1958: 15-18). Bascuñán también conoce el pensamiento administrativo estadounidense, como lo constata la referencia a las obras de White (1964), Lepawsky (1961) y Simon et al. (1956), traducidas al español y antes referidas, si bien su fuente primaria es Langrod (1954).

Con respecto al pensamiento hispanoamericano, debemos recordar sus comentarios sobre Muñoz Amato y Jiménez Castro, si bien el último es por medio

\footnotetext{
De aquí la necesidad de distinguir la persuasión y la convicción (o convencimiento), la cual, en contraste, busca el resultado de una argumentación sobre bases racionales y objetivas (Hernández Gil, 1975: 133).
} 
de un curso impartido en 1957 en Montevideo. El pensador más citado es Rafael Bielsa (1937), autor de uno de los libros sobre nuestra disciplina más relevantes. Su texto sobre la ciencia de la administración es una de las obras eximias escritas sobre la materia, mundialmente considerada.

\section{EL SITIO DE BASCUÑÁN EN LA TEORÍA DE LA ADMINISTRACIÓN PÚBLICA}

A lo largo de las páginas anteriores se han ido apuntando algunos aportes de Aníbal Bascunán al estudio de la administración pública. Esos aportes patentizan su relevancia para la disciplina y los méritos que lo sitúan, cimeramente, en ese estudio.

En primer lugar, todo apunta a que Bascuñán fue en los años de 1960 el primer pensador hispanoamericano en sustentar sin titubeos el estatuto autónomo de la administración pública. En ello fue más allá, incluso, que Muñoz Amato quien padecía una fuerte influencia estadounidense. Si su texto hubiese sido divulgado en nuestra región, se habría ahorrado mucha tinta vertida a favor de la supeditación de la administración pública a la gerencia de negocios, arropada en un concepto generalista.

En segundo lugar, él observa a la administración pública como conocimiento científico bajo un enfoque óntico donde su ser está dotado de tipicidad y es susceptible de desarrollar principios. En tercer lugar, Bascuñán sustentó vigorosamente la pertenencia de la administración pública a las ciencias sociales y las humanidades, marcando una distancia ancha y profunda con la posición tayloriana gerencial apegada a las ciencias físicas.

En fin, su obra es un libro de texto magistral, digno de colocarse en el mismo anaquel bibliográfico de obras selectas para enseñanza como el preparado por el británico E.N. Gladden, Approach to Public Administration, 1966, la Introduction a la Science Administrative del francés Bernard Gournay, publicado en 1966; y el texto del italiano Giuseppe Cataldi, Lineamenti Generali di Scienza dell'Amministrazione Pubblica (1969). Todas ellas, fueron obras publicadas en la época de Bascuñán.

La calidad del texto de Aníbal Bascuñán ha sido resaltada en las páginas precedentes, toda vez que constituye, en el idioma español, el escrito donde la ciencia de la administración pública se muestra con mayor grado de madurez. En la década de 1960 es una de las obras más avanzadas dentro de la disciplina, habida cuenta del enfoque de la administración pública como ciencia autónoma y como ciencia social; es, en suma un escrito que patentiza aquello que Waldo denominó autoconciencia de la disciplina. 


\section{REFERENCIAS}

Barnard, C. (1959). Las Funciones de los Elementos Dirigentes. Madrid: Instituto de Estudios Políticos.

Bascuñán Valdés, A. (1963). Elementos de Ciencia de la Administración Pública. Santiago: Editorial Jurídica de Chile.

Bielsa, R. (1937). Ciencia de la Administración. Rosario: Universidad Nacional del Litoral.

Burnham, J. (1943). La Revolución de los Directores. Buenos Aires: Editorial Claridad.

Cataldi, G. (1969). Lineamenti Generali di Scienza dell'Amministrazione Pubblica. Milano: Dott. A. Giuffré Editore.

Drapkin, A. (2004). El Aporte de don Aníbal Bascuñán Valdés al Estudio y Enseñanza de la Administración Pública. Estado, Gobierno, Gestión Pública. Revista Chilena de Administración Pública, II (5), 96-104.

Escoubé, P. (1958). Charles Jean Bonnin, Précurseur de la Science Administrative. La Revue Administrative, 11, 15-18.

Etzioni, A. (1965). Organizaciones Modernas. México: UTHEHA.

Finer, H. (1964). Teoría y Práctica del Gobierno Moderno. Madrid: Editorial Tecnos.

Galván Escobedo, J. (1963). Tratado de Administración General. San José, Costa Rica: Escuela Superior de Administración Pública de América Central.

Gasparri, P. (1959). La Scienza della Amministrazione. Padova: CEDAM- Casa Editrice don. Antonio Milani.

Gladden, E. N. (1966). Approach to Public Administration. London: Staples Press.

Gournay, B. (1966). Introduction a la Science Administrative. París: Presses de la Foundation Nationale des Sciences Politiques.

Gulick, L. y Urwick, L. (Eds). (1973). Ensayos sobre la Ciencia de la Administración. Madrid: Escuela Nacional de Administración Pública.

Hernández Gil, A. (1975). El Abogado y el Razonamiento Jurídico. Madrid: Editorial Rivadeneyra. 


\section{GUERRERo}

Jiménez Castro, W. (1963). Introducción al Estudio de la Teoría Administrativa. México: Fondo de Cultura Económica.

Langrod, G. (1954). La Science et l'Enseingnement de l'Administration Publique aux Etats Unis. Paris: Libraire Armand Colin.

LaPalombara, J. (Ed.). (1970). Burocracia y Desarrollo Político. Buenos Aires: Editorial Paidós.

Lepawsky, A. (1961). Administración: El Arte y la Ciencia de la Organización y Administración. México: Compañía Editora Continental.

March, J. y Simon, H. (1969). Teoría de la Organización. Barcelona: Ediciones Ariel.

Majone G. (1989). Evidence, Argument and Persuasion in the Policy Analysis. New Haven: Yale University Press.

Molitor, A. (1958). Les Sciences Sociales dans l'Enseingnement Supérieur: Administration Publique. Paris: UNESCO.

Muñoz Amato, P. (1954). Introducción a la Administración Pública. México: Fondo de Cultura Económica. Dos tomos.

Organización de Naciones Unidas (ONU) (1962). Manual de Administración Pública. Nueva York: Naciones Unidas.

Simon, H. (1964). El Comportamiento Administrativo. Madrid: Editorial Aguilar.

Simon, H. et. al. (1956). Administración Pública. San Juan: Ediciones de la Universidad de Puerto Rico.

Waldo, D. (1961). Teoría Politica de la Administración Pública. Madrid: Editorial Tecnós.

(1964). Estudio de la Administración Pública. Madrid: Aguilar.

(1967). Administración Pública. México: Editorial Trillas.

White, L. (1964). Introducción al Estudio de la Administración Pública. México: Compañía General de Ediciones.

White, J. (1999). Taking Language Seriously: The Narrative Foundations of Public Administration. Washington. Georgetown University Press.

Recibido: 15-08-2011

Aceptación de la versión final: 13-04-2012 